\title{
Severe mental illness and mortality of hospitalized ACS patients in
} the VHA

\author{
Mary E Plomondon*1, P Michael Ho 1,2, Li Wang33, Gwendolyn T Greiner3, \\ James H Shore ${ }^{4}$, Joseph T Sakai ${ }^{4}$, Stephan D Fihn ${ }^{3}$ and John S Rumsfeld ${ }^{1,2}$
}

\begin{abstract}
Address: ${ }^{1}$ Cardiology Section, Denver VA Medical Center, Denver CO, USA, ${ }^{2}$ Department of Medicine, University of Colorado Health Sciences Center, Denver CO, USA, ${ }^{3}$ Health Services Research and Development, Department of Veterans Affairs Puget Sound Health Care Center, Seattle WA, USA and ${ }^{4}$ Department of Psychiatry, University of Colorado Health Sciences Center, Denver CO, USA

Email: Mary E Plomondon* - meg.plomondon@va.gov; P Michael Ho - michael.ho@va.gov; Li Wang - li.wang2@va.gov; Gwendolyn T Greiner - gwendolyn.greiner@va.gov; James H Shore - jay.shore@uchsc.edu; Joseph T Sakai - joseph.sakai@uchsc.edu; Stephan D Fihn - stephan.fihn@va.gov; John S Rumsfeld - John.Rumsfeld@va.gov

* Corresponding author
\end{abstract}

Published: 18 September 2007

BMC Health Services Research 2007, 7:146 doi:10.1 186/1472-6963-7-146
Received: 22 June 2007

Accepted: 18 September 2007

This article is available from: http://www.biomedcentral.com//472-6963/7//46

(c) 2007 Plomondon et al; licensee BioMed Central Ltd.

This is an Open Access article distributed under the terms of the Creative Commons Attribution License (http://creativecommons.org/licenses/by/2.0), which permits unrestricted use, distribution, and reproduction in any medium, provided the original work is properly cited.

\begin{abstract}
Background: Severe mental illness (SMI) has been associated with more medical co-morbidity and less cardiovascular procedure use for older patients with myocardial infarction. However, it is unknown whether SMI is associated with increased long term mortality risk among patients presenting with acute coronary syndromes (ACS). We tested the hypothesis that SMI is associated with higher one-year mortality following ACS hospitalization.
\end{abstract}

Methods: All ACS patients $(n=14,194)$ presenting to Veterans Health Administration (VHA) hospitals between October 2003 and September 2005 were included. Survival analysis evaluated the association between SMI and one-year all-cause mortality, adjusting for demographics, comorbidities, in-hospital treatment, and discharge medications.

Results: Overall, $18.4 \%$ of ACS patients had SMI. Patients with SMI were more likely female, younger, Caucasian race, have a history of alcohol abuse, liver disease, dementia, hypertension and more likely to be a current smoker; however, prior cardiac history was similar between the 2 groups. There were no significant differences in cardiac procedure use, including coronary angiogram $(38.7 \%$ vs. $40.3 \%, p=0.14)$ or coronary revascularization $(31.0 \%$ vs. $32.3 \%, p=0.19)$, and discharge medications between those with and without SMI. One-year mortality was lower for patients with SMI $(15.8 \%$ vs. $19.1 \%, \mathrm{p}<0.00 \mathrm{I})$. However, in multivariable analysis, there were no significant differences in mortality ( $\mathrm{HR} 0.91 ; 95 \% \mathrm{Cl} \mathrm{0.8I-I.02)} \mathrm{between} \mathrm{patients} \mathrm{with} \mathrm{and} \mathrm{without}$ SMI.

Conclusion: Among ACS patients in the VHA, SMI is prevalent, affecting almost I in 5 patients. However, patients with SMI were as likely to undergo coronary revascularization and be prescribed evidence-based medications at hospital discharge, and were not at elevated risk of adverse I-year outcomes compared to patients without SMI. 


\section{Background}

Acute Coronary Syndrome (ACS), including unstable angina and acute myocardial infarction (AMI), accounts for over 800,000 hospitalizations yearly [1]. Prior studies have shown that disparities exist for AMI care according to socio-economic status, age, gender, and race. Furthermore, these disparities in AMI care have been associated with differential patient outcomes. For example, women with AMI are less likely to receive acute reperfusion therapy and have higher in-hospital mortality rates compared to men [2-7].

More recently, concerns have been raised regarding potential disparities in AMI care for patients with severe mental illness (SMI), including schizophrenia, mood disorders, anxiety disorders and personality disorders. Prior studies have found that patients with SMI were less likely to receive coronary revascularization and have higher risk of death following AMI [8-10]. The cited reasons for this inequality include increased medical comorbidity, reduced access to medical technology, social isolation, low income, interference with informed consent because of the cognitive symptoms and provider hesitation to aggressively treat SMI patients [8-10]. However, it is unknown whether this disparity exists in a fully integrated system, such as the VHA (Veterans Health Administration), where there is more equal access to medical technology.

Accordingly, the objective of this study was to evaluate the association between SMI and outcomes (1-year all-cause mortality and all-cause mortality or re-hospitalization for AMI) in a national Veterans Health Administration (VHA) cohort of ACS patients hospitalized from 2003 to 2005. We hypothesized that ACS patients with severe mental illness would undergo less coronary revascularization during the index hospitalization and have higher 1-year allcause mortality and AMI re-hospitalization. The results of this study may have important implications regarding disparities in AMI care and outcomes.

\section{Methods}

Information for this study was collected as part of the VHA External Peer Review Program (EPRP) for quality monitoring and improvement for a variety of medical conditions and procedures, including AMI and unstable angina (UA). Patients with International Classification of Diseases $9^{\text {th }}$ Revision diagnosis codes 410.xx and 411.xx were identified from the VA Patient Treatment File. Working with the EPRP abstraction contractor, West Virginia Medical Institute, the VHA Office of Quality and Performance generated a list of patients that was transmitted to VHA facilities where both paper and electronic medical records were manually abstracted by trained abstractors using standard reporting forms. Abstracted data were then entered into a database maintained by the contractor.
Additional details of the study methods have been published [11].

\section{Subjects}

All patients admitted with AMI or UA as documented by standard electrocardiographic criteria, elevated troponin levels, and/or other clinical evidence and discharged from VHA medical centers between 10/1/2003 and 9/30/2005 were included. Patients who were transferred from or to a non-VHA hospital, patients who left against medical advice and patients who died during the index hospitalization were excluded. The analytical cohort for the current study was 14,194 patients.

The primary independent predictor variable of interest was the presence of severe mental illness (SMI). Consistent with other literature, patients were categorized as having SMI if they had any one or more of the following ICD9 diagnosis codes: Schizophrenia (295.30, 295.10, 295.20, 295.90); Mood disorders (296.3X, 296.40, 296.4X, 296.6X, 296.5X, 296.7, 296.89) However, mood disorders specified as mild or either partial or full remission were not included; Anxiety disorders (300.01, 300.21,300.22, 300.23, 300.3, 309.81, 300.02); Personality disorders (301.0, 301.20, 301.22, 301.7, 301.83, 301.50, 301.81, 301.82, 301.6, 301.4, 301.9) [10].

\section{Outcome variables}

The primary outcome for this study was 1 -year all-cause mortality. The secondary outcome was the combined endpoint of all-cause mortality or re-hospitalization for AMI, within 1-year following the index ACS admission. Allcause mortality was defined using the Vital Statistics database, which combines four sources of VA mortality data; VA Beneficiary Identification and Record Locator, inpatient file, Medicare and Social Security Administration. When compared against the National Death Index, the sensitivity was over 98\% [12]. Re-hospitalization for AMI was defined using a primary discharge ICD-9 diagnosis code of 410.XX.

\section{Statistical analyses}

Baseline patient characteristics, co-morbidities, presentation factors, hospital treatment and discharge medications among eligible patients were compared between patients with and without SMI using the Chi-square test for categorical variables and t-test for continuous measures. The unadjusted survival was compared between patients with and without SMI using the Kaplan-Meier method. Survival was measured beginning at hospital discharge and censored at the time of death or re-hospitalization for AMI. Differences in event rates were evaluated with the log-rank test. Next, a series of multivariable Cox regression models were constructed to assess the association between SMI and mortality with incremental adjust- 
ment for demographic, cardiac, and non-cardiac variables, presentation variables, in-hospital procedures and discharge medications as listed in the Table 1 . The same multivariable models were constructed to assess the association between SMI and the combined outcome of all-cause mortality or re-hospitalization for AMI. Because the association between SMI and the outcomes showed no differences in significance or magnitude of effect across the incremental models, only the results of the final model are reported.

In secondary analysis, we assessed the association between SMI and outcomes among the following patient subgroups: unstable angina or acute myocardial infarction, age $\geq 65$ or $<65$, prior known coronary artery disease, and the subgoups of SMI diagnoses including, schizophrenia, mood or anxiety disorder and personality disorder.

All analyses were done using Stata version 9.0 (StataCorp, College Station, TX). This study was approved by the University of Washington Institutional Review Board and waiver of informed consent was granted.

\section{Results}

Characteristics of the study population are outlined in Table 1 . Overall, $18.4 \%(n=2,623)$ of the study population had a diagnosis of SMI. Of the patients with SMI, $65.5 \%(n=1718)$ had a diagnosis of anxiety disorder, $47.1 \%(n=1235)$ had a diagnosis of mood disorder, $15.5 \%(n=406)$ had a diagnosis of schizophrenia, and $11.7 \%(n=307)$ had a diagnosis of personality disorder (not mutually exclusive categories). Patients with SMI were younger, more often Caucasian, and more often female. They were more likely to have a diagnosis of alcohol abuse, liver disease, and dementia, and to be current smokers. Prior cardiac history was similar between the 2 groups, except that patients with SMI were less likely to have had prior CABG surgery.

Among eligible patients, there were no significant differences in the rates of receipt of diagnostic coronary angiogram and coronary revascularization between patients with and without SMI (Figure 1). At hospital discharge, there were similar prescription rates for aspirin, ACEinhibitor/ARB, and $\beta$-blocker medications between the 2 patient groups (Figure 1). In unadjusted analysis, the freedom from event rate was significantly higher among SMI

Table I: Characteristics of the study population

\begin{tabular}{|c|c|c|c|}
\hline Variables & No SMI N = I I $57 \mid(8 \mid .6 \%)$ & SMI N = 2623 (18.4\%) & p-value \\
\hline \multicolumn{4}{|l|}{ Demographic } \\
\hline Mean age (SD), yrs & $69.6(11.5)$ & $64.0(11.7)$ & $<0.001$ \\
\hline Male & 11357 (98.1\%) & $2548(97.1 \%)$ & 0.001 \\
\hline Caucasian race & $5943(51.4 \%)$ & 1553 (59.2\%) & $<0.001$ \\
\hline $\mathrm{BMI}(\mathrm{SD})$ & $27.9(5.9)$ & $28.9(6.4)$ & $<0.001$ \\
\hline \multicolumn{4}{|l|}{ Cardiac } \\
\hline Prior AMI & $2757(23.8 \%)$ & $628(23.9 \%)$ & 0.900 \\
\hline Prior $\mathrm{CHF}$ & $3259(28.2 \%)$ & $7317(27.9 \%)$ & 0.760 \\
\hline Prior CABG & $2509(21.7 \%)$ & $502(19.1 \%)$ & 0.004 \\
\hline Prior $\mathrm{PCl}(6 \mathrm{~m})$ & 487 (4.2\%) & $124(4.7 \%)$ & 0.237 \\
\hline \multicolumn{4}{|l|}{ Non-Cardiac } \\
\hline Alcohol abuse & $1037(9.0 \%)$ & $725(27.6 \%)$ & $<0.001$ \\
\hline Cancer & $1098(9.5 \%)$ & $174(6.6 \%)$ & $<0.001$ \\
\hline COPD & $2149(18.6 \%)$ & $532(20.3 \%)$ & 0.043 \\
\hline Dementia & $1016(8.8 \%)$ & 967 (36.9.0\%) & $<0.001$ \\
\hline Lipid disorder & 7327 (63.3\%) & $1655(63.1 \%)$ & 0.828 \\
\hline Liver disease & $469(4.1 \%)$ & $227(8.6 \%)$ & $<0.001$ \\
\hline Cerebral Vascular Disease & $1072(9.3 \%)$ & $242(9.2 \%)$ & 0.951 \\
\hline Musculoskeletal & $761(6.6 \%)$ & $188(7.2 \%)$ & 0.274 \\
\hline Renal disease & $2547(22.0 \%)$ & $508(19.4 \%)$ & 0.003 \\
\hline Diabetes & $2396(20.7 \%)$ & $556(21.2 \%)$ & 0.577 \\
\hline Current smokers & $3301(28.5 \%)$ & 1161 (44.3\%) & $<0.001$ \\
\hline Hypertension & $10155(87.8 \%)$ & $2355(89.8 \%)$ & 0.004 \\
\hline Peripheral Vascular Disease & $3542(30.6 \%)$ & $711(27.1 \%)$ & $<0.001$ \\
\hline \multicolumn{4}{|l|}{ Presentation } \\
\hline Heart rate & $85.6(23.0)$ & $84.9(22.0)$ & 0.130 \\
\hline Systolic blood pressure & $140(30.0)$ & $138(29)$ & 0.001 \\
\hline Diastolic blood pressure & $76(18.0)$ & 77 (19) & 0.012 \\
\hline
\end{tabular}

BMI, body mass index; AMI, myocardial infarction; CHF, chronic heart failure; COPD, Chronic obstructive pulmonary disease; CABG, coronary artery bypass graft; $\mathrm{PCl}$, percutaneous coronary intervention; COPD, Chronic obstructive pulmonary disease; Cath, Cardiac Catheterization; 


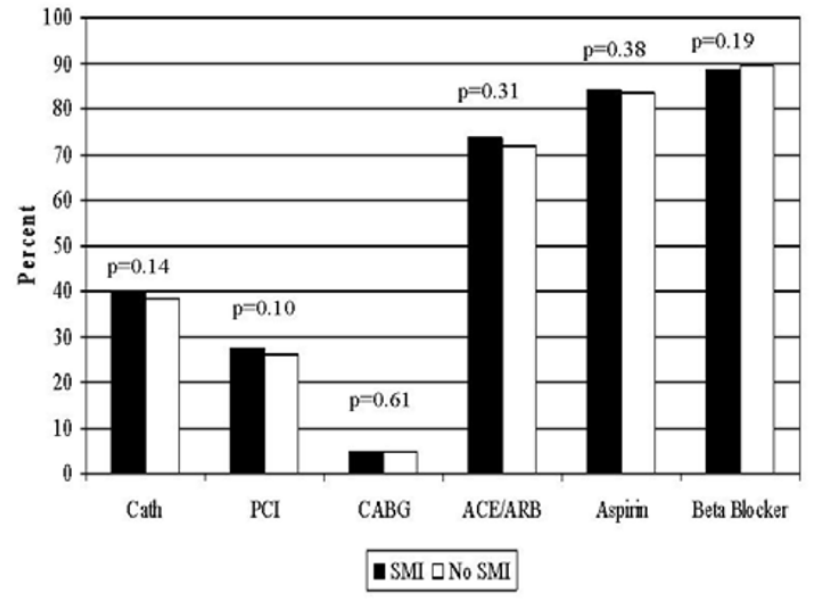

Figure I

Comparison of receipt of care SMI and non-SMI

patients. Cath = Cardiac Catheterization $; \mathrm{PCl}=$ percutaneous coronary intervention; $C A B G=$ coronary artery bypass graft; $A C E I / A R B$ = angiotensin-converting enzyme inhibitors/ Angiotensin II Receptor Blockers;

patients compared to those without SMI for 1-year allcause mortality ( $84.2 \%$ vs $80.9 \%, \mathrm{p}<0.01$ ) and the combined endpoint of all-cause mortality and re-hospitalization (78.0\% vs 75.6\%, p = 0.01) for AMI (Figures 2 and 3).

However, after adjustment for demographic, cardiac and non-cardiac co-morbidities, presentation factors, in-hospital procedures and discharge medications, there was no significant association between SMI and all-cause mortality (HR $0.91 ; 95 \%$ CI $0.81-1.02$ ) or the combined endpoint of all-cause mortality and re-hospitalization for AMI, (HR 0.99; 95\% 0.90-1.10). The findings were con-

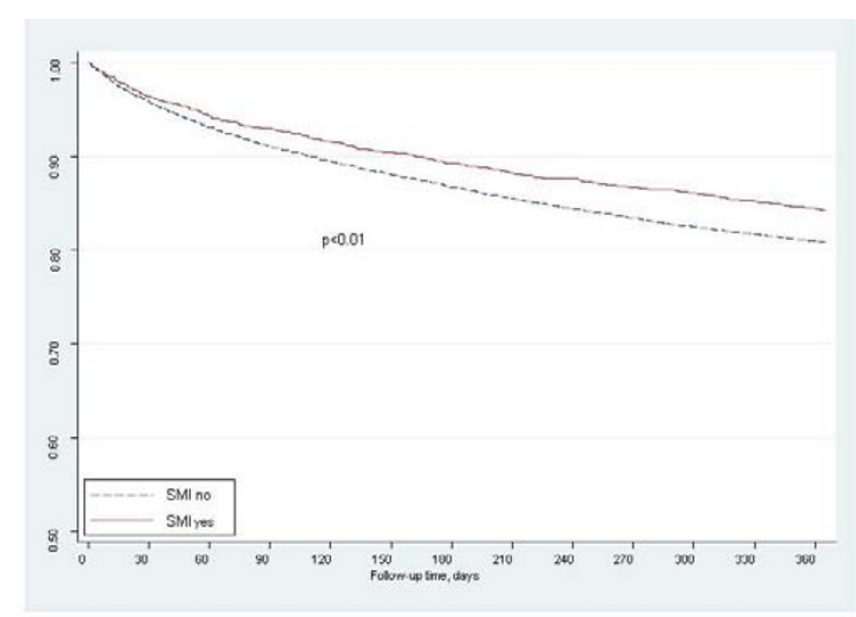

Figure 2

Freedom from I-year mortality.

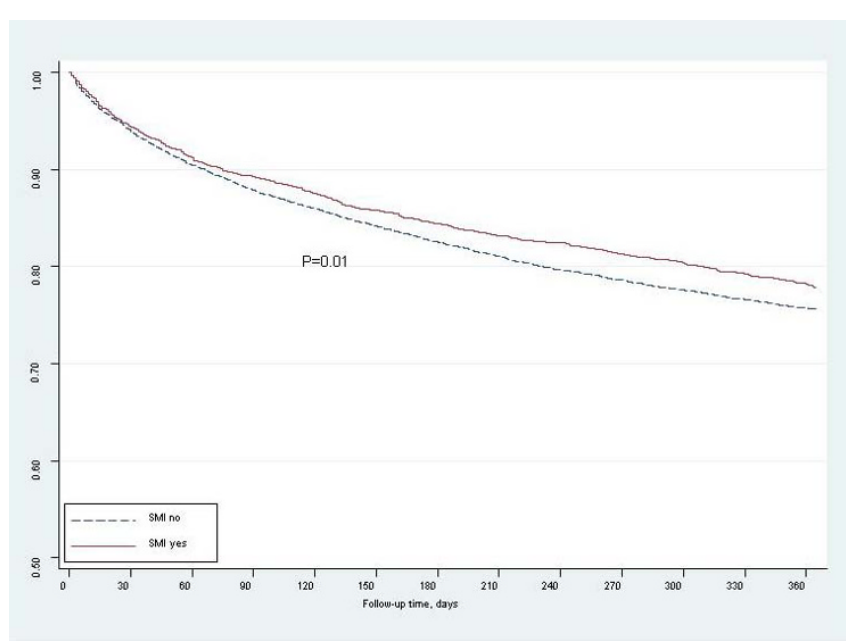

Figure 3

Freedom from I-year mortality/re-hospitalization.

sistent when analyzed by subgroups of SMI ; schizophrenia $(0.83 ; 95 \%$ CI 0.60-1.15), mood/anxiety disorder (0.91; 95\% CI 0.79-1.06), and personality disorder (1.0; 95\% CI 0.68-1.5). Additionally, the association between SMI and outcomes remained consistent when analyzed by different patient subgroups, including unstable angina or myocardial infarction, age $\geq 65$ or $<65$, and prior known coronary artery disease.

\section{Discussion}

The goal of this study was to assess the association between SMI and 1-year all-cause mortality and AMI rehospitalization among a national cohort of veterans with ACS. Overall, $18.4 \%$ of the study population had a diagnosis of SMI. There were no significant differences in the rates of coronary angiography, PCI or CABG during the index hospitalization or prescription rates of evidencebased discharge medications between patients with and without SMI. Finally, there were no significant differences in risk-adjusted 1-year all-cause mortality or AMI re-hospitalization between ACS patients with and without SMI.

The existing literature on the association between SMI and outcomes following acute myocardial infarction has been mixed. In prior studies, the prevalence of SMI among patients presenting with AMI ranged from $4.7 \%$ to $40 \%$ depending on the population studied and the definitions used for SMI. While other studies have had a broader definition of SMI and included diagnoses such as other psychoses, alcohol/substance abuse disorders, and/or adjustment reaction as part of their definition, we had a more restricted definition and only included patients with schizophrenia, mood disorders, anxiety disorders and personality disorders $[8-10,13,14]$. In addition, previous studies have shown that patients with severe mental ill- 
ness were less likely to undergo diagnostic coronary angiography, and revascularization procedures and had a trend towards higher 1 year mortality following AMI hospitalization [8-10,14]. In contrast, we assessed a contemporary cohort of ACS patients with SMI in a large integrated health care system, used chart abstracted data for risk-adjustment, and performed sensitivity analyses among strata of SMI and patient subgroups. We found that there were no significant differences in the rates of coronary angiography and coronary revascularization between eligible patients with and without SMI and 1year all-cause mortality and AMI re-hospitalization. These findings suggest that the presence of SMI among patients presenting with ACS does not negatively impact in-hospital care or long-term outcomes in the VHA.

There are several potential explanations for our findings of no difference in outcomes between patients with or without SMI presenting with ACS in the VHA. First, access to care may be less of an issue for SMI patients in the VHA compared to other healthcare settings. Other studies of patients in the VHA have shown no disparities of care and outcomes by race and gender $[15,16]$. Second, a prior study found that VA physicians were more likely to initiate mental health referral for patients with depression compared to non-VA physicians [17]. One potential explanation is that mental health disorders are common among veterans and because it is a common condition, there may be less of a physician bias in the VHA compared to non-VHA settings. Finally, prior studies have shown that a collaborative care model between primary care and mental health providers for VHA patients with depression is associated with more rapid improvement in symptoms as well as mental health status [18,19]. Additional studies are needed to determine if such a care model for patients with concomitant cardiovascular and severe mental illness will lead to improved patient outcomes.

There are several limitations of this study which should be acknowledged. The results of this study may not be generalizable to all patients with SMI who are hospitalized for ACS because our study cohort comprised of veterans who were predominantly male, older, and had significant comorbidities. Additionally, we were unable to adjust for the veterans socioeconomic status. However, VHA eligibility is determined by disability related to military service or by economic disadvantage [20]. This study included a national cohort of all patients with ACS in the VHA from 2003 through 2005 and therefore represents practice patterns for patients with SMI and ACS in a large integrated healthcare system. Second, we defined SMI using ICD-9 codes and, therefore, could not assess the severity of a patients' mental illness and future studies should assess whether patient outcomes following ACS hospitalization vary according to the severity of the underlying mental disorder. Third, we did not observe a difference in 1-year outcomes following ACS hospitalization between patients with and without SMI, raising the possibility of Type II error. However, we had $99 \%$ power to detect an absolute mortality difference of $3.5 \%$ between the 2 comparison groups.

\section{Conclusion}

In summary, we found that among ACS patients in the VHA, SMI is prevalent affecting almost 1 in 5 ACS patients. However, patients with SMI were just as likely to undergo coronary revascularization and to be prescribed evidence-based medications at hospital discharge compared to patients without SMI. One-year mortality and rehospitalization for MI were similar between the two groups. Thus, there is no evidence for disparity in care and outcomes based on the presence of SMI among ACS patients in the VHA.

\section{Abbreviations}

SMI, severe mental illness; ACS, acute coronary syndrome; VHA, Veterans Health Administration; AMI, acute myocardial infarction; EPRP, external peer review program; US, unstable angina; $\mathrm{BMI}$, body mass index; $\mathrm{CHF}$, chronic heart failure; COPD, Chronic obstructive pulmonary disease; CABG, coronary artery bypass graft; $\mathrm{PCI}$, percutaneous coronary intervention; COPD, Chronic obstructive pulmonary disease; Cath, Cardiac Catheterization;

\section{Competing interests}

The author(s) declare that they have no competing interests.

\section{Authors' contributions}

All authors made substantial contributions to conception and design, or acquisition of data, or analysis and interpretation of data. All authors have been involved in drafting of manuscript or revising it critically for important intellectual content; and all authors have given final approval of the version to be published.

\section{Acknowledgements}

This work was supported by a grant from the Department of Veterans Affairs Ischemic Heart Disease Quality Enhancement Research Initiative. The views expressed in this article are those of the authors and do not necessarily represent the views of the Department of Veterans Affairs.

\section{References}

I. American Heart Association: Heart Disease and Stroke Statistics - 2006 Update. 2006. Ref Type: Report

2. Blomkalns AL, Chen AY, Hochman JS, Peterson ED, Trynosky K, Diercks DB, et al:: Gender disparities in the diagnosis and treatment of non-ST-segment elevation acute coronary syndromes: large-scale observations from the CRUSADE (Can Rapid Risk Stratification of Unstable Angina Patients Suppress Adverse Outcomes With Early Implementation of the American College of Cardiology/American Heart Association Guidelines) National Quality Improvement Initiative. J Am Coll Cardiol 2005, 45:832-837. 
3. Ayanian JZ, Epstein AM: Differences in the use of procedures between women and men hospitalized for coronary heart disease. N Engl J Med I99I, 325:22 I-225.

4. Milcent C, Dormont B, Durand-Zaleski I, Steg PG: Gender differences in hospital mortality and use of percutaneous coronary intervention in acute myocardial infarction: microsimulation analysis of the 1999 nationwide French hospitals database. Circulation 2007, I I 5:833-839.

5. Epstein AM, Weissman JS, Schneider EC, Gatsonis C, Leape LL, Piana RN: Race and gender disparities in rates of cardiac revascularization: do they reflect appropriate use of procedures or problems in quality of care? Med Care 2003, 4I:1240-1 255.

6. Rao SV, Kaul P, Newby LK, Lincoff AM, Hochman J, Harrington RA, et al:: Poverty, process of care, and outcome in acute coronary syndromes. J Am Coll Cardiol 2003, 4I: I948- 1954.

7. Vaccarino V, Rathore SS, Wenger NK, Frederick PD, Abramson JL, Barron HV, et al.: Sex and racial differences in the management of acute myocardial infarction, 1994 through 2002. N Engl J Med 2005, 353:67I-682.

8. Lawrence DM, Holman CD, Jablensky AV, Hobbs MS: Death rate from ischaemic heart disease in Western Australian psychiatric patients 1980-1998. Br J Psychiatry 2003, 182:3I-36.

9. Kisely S, Smith M, Lawrence D, Cox M, Campbell LA, Maaten S: Inequitable access for mentally ill patients to some medically necessary procedures. CMAJ 2007, 176:779-784.

10. Druss BG, Bradford DW, Rosenheck RA, Radford MJ, Krumholz HM Mental disorders and use of cardiovascular procedures after myocardial infarction. JAMA 2000, 283:506-5II.

II. Maynard C, Lowy E, Rumsfeld JS, Sales AE, Sun H, Kopjar B, et al.: The Prevalence and Outcomes of In-Hospital Acute Myocardial Infarction in the Department of Veterans Affairs Health System. Arch Intern Med 2006, 166:1410-1416.

12. Sohn MW, Arnold N, Maynard C, Hynes DM: Accuracy and completeness of mortality data in the Department of Veterans Affairs. Popul Health Metr 2006, 4:2.

13. Jones LE, Carney CP: Mental disorders and revascularization procedures in a commercially insured sample. Psychosom Med 2005, 67:568-576.

14. Petersen LA, Normand SL, Druss BG, Rosenheck RA: Process of care and outcome after acute myocardial infarction for patients with mental illness in the VA health care system: are there disparities? Health Serv Res 2003, 38:4I-63.

15. Jha AK, Perlin JB, Steinman MA, Peabody JW, Ayanian JZ: Quality of ambulatory care for women and men in the Veterans Affairs Health Care System. J Gen Intern Med 2005, 20:762-765.

16. Rumsfeld JS, Plomondon ME, Peterson ED, Shlipak MG, Maynard C, Grunwald GK, et al.: The impact of ethnicity on outcomes following coronary artery bypass graft surgery in the Veterans Health Administration. J Am Coll Cardiol 2002, 40: I 786- 1793.

17. Frayne SM, Freund KM, Skinner KM, Ash AS, Moskowitz MA: Depression management in medical clinics: does healthcare sector make a difference? Am J Med Qual 2004, 19:28-36.

18. Kilbourne AM, Pincus HA, Schutte K, Kirchner JE, Haas GL, Yano EM: Management of mental disorders in VA primary care practices. Adm Policy Ment Health 2006, 33:208-2I4.

19. Bauer MS, McBride L, Williford WO, Glick H, Kinosian B, Altshuler L, et al.: Collaborative care for bipolar disorder: Part II. Impact on clinical outcome, function, and costs. Psychiatr Serv 2006 , 57:937-945.

20. Petersen LA, Normand SL, Daley J, McNeil BJ: Outcome of myocardial infarction in Veterans Health Administration patients as compared with medicare patients. N Engl J Med 2000, 343:|934-|94|.

\section{Pre-publication history}

The pre-publication history for this paper can be accessed here:

http://www.biomedcentral.com/1472-6963/7/146/pre pub
Publish with Biomed Central and every scientist can read your work free of charge

"BioMed Central will be the most significant development for disseminating the results of biomedical research in our lifetime. "

Sir Paul Nurse, Cancer Research UK

Your research papers will be:

- available free of charge to the entire biomedical community

- peer reviewed and published immediately upon acceptance

- cited in PubMed and archived on PubMed Central

- yours - you keep the copyright
BioMedcentral 